\title{
Section introduction \\ Are current drug development programmes realising the full potential of new agents? Introduction to Sessions 7 and 8
}

\author{
Stephen RD Johnston
}

Department of Medicine, Royal Marsden NHS Foundation Trust, Chelsea, London SW3 6JJ, UK

Stephen RD Johnston, stephen.johnston@rmh.nhs.uk

Published: 18 December 2009

This article is online at

http://breast-cancer-research.com/supplements/11/S3/S20

The final two sessions of the meeting addressed the question of whether current drug development programmes for new agents in breast cancer are realising their full potential.

Dr Stephen Johnston gave an overview of the scenario and outlined how, in an era of molecular pathology and targeted therapies, the challenges facing both academic investigators and the pharmaceutical industry are indeed significant. Despite the preclinical promise that many novel therapeutics have shown, translating that promise into significant patient benefit does not always happen. The traditional clinical development pathway where one size fits all no longer applies to modern targeted therapies, as exemplified by the lessons we have learnt form HER2 targeting with both trastuzumab and, more recently, lapatinib. Combination strategies that address the complexity of the integrative networks on which these molecular targets impact are now emerging.

Dr Robert Clarke examined some of the principles behind the preclinical development that are needed to bring a new molecule from early discovery and synthesis through to firstin-man clinical studies. As the human genome is unravelled, the major challenge faced by scientists is the multitude of at least 500 drug targets in cancer, and the vast complex of interdependent networks on which each target impacts. Compensatory pathways that become operative when a given target is blocked can severely limit the development of a potent inhibitor of what seems like a very suitable oncogenic target. As such, effective combinations are much more likely to be effective than individual targeted drugs, and early assessment of safety and toxicity in preclinical in vivo models will remain necessary. Efficacy testing of these drugs in xenograft models, however, does not always reflect the heterogeneity of human breast cancer - and the concept of early phase zero trials as proof of concept may be an effective way to anticipate failure and to reject ineffective drugs before larger scale clinical development is triggered.
Breast Cancer Research 2009, 11(Suppl 3):S20

(doi:10.1186/bcr2439)

(c) 2009 BioMed Central Ltd

Professor David Cameron discussed some of the principles of founder clinical trials in breast cancer, and challenged some of the current thinking around the design of phase II/III trials for novel targeted therapies. The key dilemma lies between selecting patients for a novel drug based on some predefined clinical criteria or molecular biomarker in the tumour, or treating all comers and powering the trial for benefit in predefined stratified groups. While the former has a higher likelihood of success, recruitment may be slower and clinical benefit in other groups could be missed. On the other hand, the larger more pragmatic clinical trial remains expensive and a higher risk, yet may yield otherwise unknown information about the benefit of a new therapy in certain groups of patients.

Professor John Robertson described the development of small molecule tyrosine kinase inhibitors targeted against the epidermal growth factor receptor in breast cancer. The preclinical rationale was strong - namely that epidermal growth factor receptor expression was enhanced in models of acquired endocrine resistance and that gefitinib may be effective in tamoxifen-resistant disease, or when combined with endocrine therapy to delay development of acquired resistance. While the efficacy of gefitinib in monotherapy studies in advanced disease was disappointing, neoadjuvant presurgical studies with both gefitinib and erlotinib demonstrated clear biological effects with the drugs in oestrogen receptor-positive breast cancer. The most recent clinical trials of endocrine therapy combined with gefitinib were reviewed. Appropriate target identification and selection have limited the successful development of epidermal growth factor receptor inhibitors, and while activating mutations have proved crucial in understanding benefit in lung cancer, the same has never been demonstrated in breast cancer.

Dr Serena Di Cosimo discussed the emerging data regarding mammalian target of rapamycin (mTOR) antagonists, and the

$\mathrm{mTOR}=$ mammalian target of rapamycin. 
phosphatidylinositol-3-kinase/Akt pathway in particular, as a viable target in breast cancer. Promising preclinical data demonstrated that blockade of this target in oestrogen receptor-positive breast cancer could enhance endocrine responsiveness, which supported the development of clinical trials in breast cancer combining aromatase inhibitors with mTOR antagonists - while a large-scale phase III trial in metastatic disease was negative, a preoperative neoadjuvant study with detailed biomarker analyses identified added benefit in tumours with activating PI3CA exon 9 mutations. Furthermore, understanding that $\mathrm{mTOR}$ antagonism released an important negative feedback loop that then activated Akt via insulin-like growth factor receptor substrate 1 has led to new combination strategies emerging - in particular, using an insulin-like growth factor-1 receptor antibody in addition to an mTOR antagonist. As such, mTOR blockade could still be an important strategy in breast cancer once the most effective combinations have been developed.

There then followed an open forum and discussion session in which the speakers were joined by three senior representatives from the pharmaceutical industry (Maria Koehler, lan C Smith, Ajay Bhatnagar), all of whom have been involved in development of novel therapies for breast cancer. The challenges faced by the industry were debated, including the complex issue of how to prioritise development of molecules in a scenario where numerous targets and potential lead compounds now exist. Approaches to clinical trial design that may allow the most effective agents to be identified early were discussed, in addition to how modern platforms that provide a molecular profile of human breast cancer could be integrated into early-phase clinical development. Collaboration between the pharmaceutical industry, academic institutions and clinical investigators will clearly remain crucial if the new era of targeted therapies is to be translated into significant gains in clinical outcome for women with breast cancer.

The session concluded with a plenary lecture from Dr Dennis Slamon, who outlined the lessons we had learnt from the identification and successful targeting of the HER2 oncogene in breast cancer. Central to this was the ability to measure the target in human breast cancer appropriately and to select the patients who would derive the most benefit. He outlined some of the current challenges that we face in assay development for any new target, and how molecular pathology needs to be integrated into early-phase trial design in order to maximise the potential of the numerous new agents that are now available for clinical investigation.

\section{Competing interests}

The author declares that they have no competing interests.

\section{Acknowledgement}

This article has been published as part of Breast Cancer Research Volume 11 Suppl 3 2009: Controversies in Breast Cancer 2009. The full contents of the supplement are available online at http://breastcancer-research.com/supplements/11/S3. 